Revista de MAtemática: Teoría y APliCACiOnes 2015 22(1) : 89-112

CIMPA - UCR ISSN: 1409-2433 (PRINT), 2215-3373 (ONLINE)

\title{
OPTIMAL PRODUCTION-SALES STRATEGIES FOR A COMPANY AT CHANGING MARKET PRICE
}

\author{
ESTRATEGIAS ÓPTIMAS DE VENTAS Y \\ PRODUCCIÓN PARA UNA COMPAÑÍA EN UN \\ MERCADO CON PRECIOS CAMBIANTES
}

ELLINA V. GRIGORIEVA* EVGENII N. KHAILOV ${ }^{\dagger}$

Received: 23/Feb/2014; Revised: 28/Aug/2014;

Accepted: $3 /$ Oct/2014

\footnotetext{
*Department of Mathematics and Computer Sciences, Texas Woman's University, Denton, TX 76204, USA. E-Mail: egrigorieva@mail.twu.edu

${ }^{\dagger}$ Department of Computational Mathematics and Cybernetics, Moscow State Lomonosov University, Moscow, 119992, Russia. E-Mail: khailov@cs.msu.su
} 


\begin{abstract}
In this paper we consider a monopoly producing a consumer good of high demand. Its market price depends on the volume of the produced goods described by the Cobb-Douglas production function. A productionsales activity of the firm is modeled by a nonlinear differential equation with two bounded controls: the share of the profit obtained from sales that the company reinvests into expanding own production, and the amount of short-term loans taken from a bank for the same purpose. The problem of maximizing discounted total profit on a given time interval is stated and solved. In order to find the optimal production and sales strategies for the company, the Pontryagin maximum principle is used. In order to investigate the arising two-point boundary value problem for the maximum principle, an analysis of the corresponding Hamiltonian system is applied. Based on a qualitative analysis of this system, we found that depending on the initial conditions and parameters of the model, both, singular and bangbang controls can be optimal. Economic analysis of the optimal solutions is discussed.
\end{abstract}

Keywords: nonlinear microeconomic control model; production-sales strategy; Pontryagin maximum principle; Hamiltonian system.

\title{
Resumen
}

En este artículo consideramos un monopolio produciendo un producto de consumo de gran demanda. Su precio de mercado depende del volumen de producción descrito por la función de producción de Cobb-Douglas. Una actividad de producción y ventas de la firma es modelada por una ecuación diferencial no lineal con dos controles de frontera: la participación en el resultado de las ventas que la compañía reinvierte para expandir su propia producción, y el monto de los préstamos a corto plazo adquiridos del sistema bancario con el mismo propósito. Se plantea y resuelve el problema de maximizar la ganancia total descontada en un intervalo de tiempo dado. Para encontrar las estrategias óptimas de producción y ventas para la compañía, se usa el principio del máximo de Pontryagin. Para investigar el problema de valores de dos puntos de frontera que aparece para el principio del máximo, se aplica un análisis del sistema hamiltoniano correspondiente. Basado en un análisis cualitativo del sistema, encontramos que dependiendo de las condiciones iniciales y los parámetros del modelo, tanto el control singular como el bang-bang pueden ser óptimos. Se discute un análisis económico de las soluciones óptimas.

Palabras clave: modelo de control microeconómico no lineal; estrategia de producción y ventas; principio del máximo de Pontryagin; sistema hamiltoniano.

Mathematics Subject Classification: 49J15, 58E25, 90A16, 93B03. 


\section{Introduction}

Behavior of economic processes can be understood and predicted using mathematical models. Many well-known multidimensional microeconomic models such as the Leontief model [11] or the Loon's model [12] are linear. While nonlinearity characterizes most of economic processes and phenomena, analytical study of multi-dimensional nonlinear models is rather complex and laborious. Of course, one can experiment with such models on a computer, but an analytical study of an economic model compared with simulation of the model on a computer has an important advantage. It gives us the opportunity to get the whole picture of the studied process or phenomenon for any values of the model's parameters, while the simulation gives only a certain fragments of the general picture at the selected parameter values. Therefore, in order to study the long-term trends, growth factors or assess the impact of various options for management decisions, the study of the lower-sector nonlinear models are applied. One such model is the one-sector Solow-Swan model $[15,16]$. In this model, the company produces a single product, and sells it to obtain profit. The profit from sales can be used for both, consumption and investment. The ability to manage such capital distribution leads to the consideration of various control models, as well as to the study of the corresponding optimal control problems $[1,7,3,4,5,8]$. In this paper, the economic dynamics of a company is described by the SolowSwan type control model. What differs our work from the papers referred earlier is that the market (sales) price of the produced good depends on a certain model of the market. In Section 2 we introduce our control model alone with the model-driven mechanism for the formation of the market price of the product; the properties of the model are also studied. Additionally, in this section we state the corresponding optimal control problem and discuss the existence of its optimal solutions. In order to analyze the optimal control problem, in Section 3, we apply the Pontryagin maximum principle and state the corresponding two-point boundary value problem for the maximum principle. In Section 4 we study the possibility for the boundary value problem to have singular regimes. Section 5 contains auxiliary results related to the subsequent analysis of the Hamiltonian system, which is issued based on the boundary value problem of the maximum principle. Section 6 presents an analysis of the Hamiltonian system, based on the results obtained in the previous section. This analysis allows us to specify in Section 7 all possible types of the optimal controls that can arise in the study of the optimal control problem. In Section 8, we describe the numerical procedure that helps us to investigate the cases for which we cannot predict analytically specific features of the optimal control, but only its type. Finally, in Section 9 we describe possible directions for further research related to modification of the 
control model so as to the corresponding optimal control problem. Section 10 contains our conclusions.

\section{Optimal control problem}

Consider a company that produces a single consumer good of high demand on a stable market. We assume that the company is a monopoly in this market. Moreover, we suppose that the company sells all it produces at the market price. Let $x$ be the production funds of the company. Then, $Y=\Pi(x)$ is the amount of the produced consumer good, where $\Pi(x)$ is the Cobb-Douglas production function given by the formula $\Pi(x)=r x_{0}^{1-\sigma} x^{\sigma}, \sigma \in(0,1]$. Here $x_{0}$ is the initial production funds, and $r$ is the profitability of the production with the initial production funds of $x_{0}$.

Let $p$ be the market price of the produced consumer good, and $p_{0}$ be its unit price. We assume that the dynamics of the production funds of the company obeys the Cauchy problem

$$
\left\{\begin{array}{l}
\dot{x}(t)=I(t)-\delta x(t), \quad t \in[0, T], \\
x(0)=x_{0}, x_{0}>0 .
\end{array}\right.
$$

where $\delta$ is amortization factor that shows depreciation of the production funds, and $I(t)$ is total investments made by the company to increase the production funds.

The main balance relationship at time $t \in[0, T]$ is as follows

$$
p(t) Y(t)=I_{1}(t)+C(t),
$$

where the left hand side is the total profit obtained from the market sale of the consumer good in the amount of $Y(t)$ at the price $p(t)$. The right hand side there is the sum of the own investments $I_{1}(t)$ and consumption $C(t)$. We suppose that the total investment $I(t)$ consists of the own investment $I_{1}(t)$ and of the outside investment $I_{2}(t)$. The own investment $I_{1}(t)$ is the share of the profit $u(t) p(t) Y(t)$, received from the sales of the consumer good on the market. Investment $I_{2}(t)$ is the short-term loans $K(t)$. Here the functions $u(t), K(t)$ are controls, which satisfy the restrictions:

$$
0 \leq u(t) \leq 1, \quad 0 \leq K(t) \leq K_{\max }
$$

From the formula (2) and previous arguments it is easy to see that $C(t)=(1-$ $u(t)) p(t) Y(t)$. 
By the definition of the function $Y(t)$ and the equation (1), we have the Cauchy problem

$$
\left\{\begin{array}{l}
\dot{x}(t)=u(t) p(t) \Pi(x(t))+K(t)-\delta x(t), \quad t \in[0, T] \\
x(0)=x_{0}, x_{0}>0 .
\end{array}\right.
$$

By the model of the market [18], we assume that the market price of the consumer good satisfies the following formula

$$
p(t)=\bar{p}-b Y(t)=\bar{p}-b \Pi(x(t))>0, \quad t \in[0, T],
$$

where $\bar{p}$ is the maximum possible market price of the consumer good, and $b$ is some given constant. Substituting the expression (5) into the differential equation of the Cauchy problem (4), we rewrite it as follows

$$
\left\{\begin{array}{l}
\dot{x}(t)=u(t)(\bar{p}-b \Pi(x(t))) \Pi(x(t))+K(t)-\delta x(t), \quad t \in[0, T], \\
x(0)=x_{0}, x_{0}>0 .
\end{array}\right.
$$

As the objective function we choose the total discounted profit of the company on the given time interval $[0, T]$ of the following type

$$
\begin{gathered}
I(u, K)=\int_{0}^{T} \mathrm{e}^{-\rho t}\left\{(1-u(t))(\bar{p}-b \Pi(x(t))) \Pi(x(t))-p_{0} \Pi(x(t))\right. \\
-(1+\lambda) K(t)\} d t+\mathrm{e}^{-\rho T} x(T) .
\end{gathered}
$$

It is required to maximize this objective function on the set of admissible controls, which consists of all possible Lebesgue measurable functions $(u(t), K(t))$ satisfying the inequalities (3) for almost all $t \in[0, T]$. In the expression (7), the value $\rho$ is the discounting coefficient that reflects the rate of inflation, and $\lambda$ is the loan annual percentage rate. The term $\mathrm{e}^{-\rho T} x(T)$ is the discounted production funds of the company at the final time $T$. Moreover, the solution $x(t)$ must be positive and satisfy the inequality from (5) for all $t \in[0, T]$. Thus, we have the optimal control problem (7) subject to (6) with controls satisfying (3).

Next, we consider the case of a linear Cobb-Douglas production function, i.e. $\sigma=1$. Then, the Cauchy problem (6) can be written as

$$
\left\{\begin{array}{l}
\dot{x}(t)=u(t)(\bar{p}-b r x(t)) r x(t)+K(t)-\delta x(t), \quad t \in[0, T], \\
x(0)=x_{0}, x_{0}>0,
\end{array}\right.
$$

and the objective function (7) is transformed as follows

$$
\begin{gathered}
I(u, K)=\int_{0}^{T} \mathrm{e}^{-\rho t}\left\{(1-u(t))(\bar{p}-b r x(t)) r x(t)-p_{0} r x(t)\right. \\
-(1+\lambda) K(t)\} d t+\mathrm{e}^{-\rho T} x(T) .
\end{gathered}
$$


In order to simplify the optimal control problem (3), (8), (9) we introduce a new variable $y=b r \bar{p}^{-1} x$, and also following positive constants $\alpha=\bar{p} r$, $\beta=p_{0} r$, where $\alpha>\beta$. We also define a new control $v(t)=b r \bar{p}^{-1} K(t)$, where $0 \leq v(t) \leq v_{\max }$ and $v_{\max }=b r \bar{p}^{-1} K_{\max }$. At last, let $y_{0}=b r \bar{p}{ }^{-1} x_{0}$. Moreover, the positivity of the price $p(t)$ in the formula (5) consists of satisfying the condition $y(t)<1$ for all $t \in[0, T]$. Then, the Cauchy problem (8) and the objective function (9) we rewrite as

$$
\begin{gathered}
\left\{\begin{array}{c}
\dot{y}(t)=\alpha u(t)(1-y(t)) y(t)+v(t)-\delta y(t), \quad t \in[0, T], \\
y(0)=y_{0}, y_{0} \in(0,1),
\end{array}\right. \\
I(u, v)=\frac{\bar{p}}{b r}\left(\int_{0}^{T} \mathrm{e}^{-\rho t}\{\alpha(1-u(t))(1-y(t)) y(t)-\beta y(t)\right. \\
\left.-(1+\lambda) v(t)\} d t+\mathrm{e}^{-\rho T} y(T)\right) .
\end{gathered}
$$

By the class of admissible controls $\Omega(T)$ we understand all possible Lebesgue measurable functions $(u(t), v(t))$ that for almost all $t \in[0, T]$ satisfy the restrictions:

$$
0 \leq u(t) \leq 1, \quad 0 \leq v(t) \leq v_{\max } .
$$

For convenience, we introduce the new objective function

$$
\begin{gathered}
J(u, v)=\int_{0}^{T} \mathrm{e}^{-\rho t}\{\alpha(1-u(t))(1-y(t)) y(t)-\beta y(t) \\
-(1+\lambda) v(t)\} d t+\mathrm{e}^{-\rho T} y(T),
\end{gathered}
$$

which is different from the function (11) by the positive factor $\bar{p}(b r)^{-1}$. Maximizing this objective function on the set $\Omega(T)$ of all admissible controls, we will consider further. Moreover, the solution $y(t)$ must satisfy the inequalities:

$$
0<y(t)<1, \quad t \in[0, T] .
$$

Thus, we obtain the optimal control problem (10), (12)-(14).

Now, we discuss the execution of the restrictions (14). Suppose that in the subsequent arguments the inequality $\delta>v_{\max }$ is valid. It means that the amount of the loan $K(t)$ is comparable to the amount $\delta x(t)$ of production funds outgoing from productive activities. Note that if the right inequality in (14) is violated, then it can lead to the excessive filling of the market by the consumer good and reduce its market price to zero, which is impossible. Therefore, following statement holds. 
Lemma 1 For arbitrary controls $(u(\cdot), v(\cdot)) \in \Omega(T)$ the solution $y(t)$ of the corresponding Cauchy problem (10) exists on the entire interval $[0, T]$ and satisfies the inequalities (14).

By Lemma 1 and results from [10], we see that in the problem (10), (12)-(14) there exists the optimal decision consisting of the optimal controls $\left(u_{*}(t), v_{*}(t)\right)$ and corresponding optimal solution $y_{*}(t), t \in[0, T]$. For their analysis we apply the Pontryagin maximum principle [13]. The results of this analysis are presented in subsequent sections.

\section{Pontryagin maximum principle}

We write the Hamiltonian of the problem (10), (12)-(14) as

$H(y, \psi, u, v, t)=(\alpha u(1-y) y+v-\delta y) \psi+\mathrm{e}^{-\rho t}(\alpha(1-u)(1-y) y-\beta y-(1+\lambda) v)$,

where $\psi$ is the adjoint variable.

Then, by the virtue of the Pontryagin maximum principle [13], for the optimal controls $\left(u_{*}(t), v_{*}(t)\right)$ and corresponding optimal solution $y_{*}(t)$ there exits the nontrivial solution $\psi_{*}(t)$ of the adjoint system

$$
\left\{\begin{aligned}
\dot{\psi}_{*}(t)= & -\left(\alpha u_{*}(t)\left(1-2 y_{*}(t)\right)-\delta\right) \psi_{*}(t) \\
& -\mathrm{e}^{-\rho t}\left(\alpha\left(1-u_{*}(t)\right)\left(1-2 y_{*}(t)\right)-\beta\right), \quad t \in[0, T], \\
\psi_{*}(T)= & \mathrm{e}^{-\rho T}
\end{aligned}\right.
$$

such that the controls $\left(u_{*}(t), v_{*}(t)\right)$ maximize the Hamiltonian $H\left(y_{*}(t), \psi_{*}(t), u, v, t\right)$ with respect to the values $u, v$ for almost all $t \in[0, T]$, and therefore satisfy the relationships:

$$
\begin{gathered}
u_{*}(t)=\left\{\begin{array}{lll}
0, & \text { if } & \alpha\left(1-y_{*}(t)\right) y_{*}(t)\left(\psi_{*}(t)-\mathrm{e}^{-\rho t}\right)<0, \\
\forall u \in[0,1], & \text { if } & \alpha\left(1-y_{*}(t)\right) y_{*}(t)\left(\psi_{*}(t)-\mathrm{e}^{-\rho t}\right)=0, \\
1, & \text { if } & \alpha\left(1-y_{*}(t)\right) y_{*}(t)\left(\psi_{*}(t)-\mathrm{e}^{-\rho t}\right)>0,
\end{array}\right. \\
v_{*}(t)=\left\{\begin{array}{lll}
0, & \text { if } & \psi_{*}(t)-(1+\lambda) \mathrm{e}^{-\rho t}<0, \\
\forall v \in\left[0, v_{\max }\right], & \text { if } & \psi_{*}(t)-(1+\lambda) \mathrm{e}^{-\rho t}=0, \\
v_{\max }, & \text { if } & \psi_{*}(t)-(1+\lambda) \mathrm{e}^{-\rho t}>0 .
\end{array}\right.
\end{gathered}
$$


Now, in these relationships and in the system (15), we will execute the substitution of the variable $\eta_{*}(t)=\mathrm{e}^{\rho t} \psi_{*}(t)-1$. Then, the adjoint system (15) can be rewritten as

$$
\left\{\begin{aligned}
\dot{\eta}_{*}(t)= & -\left(\alpha u_{*}(t)\left(1-2 y_{*}(t)\right)-(\delta+\rho)\right) \eta_{*}(t) \\
& -\left(\alpha\left(1-2 y_{*}(t)\right)-(\delta+\rho+\beta)\right), \quad t \in[0, T], \\
\eta_{*}(T)= & 0
\end{aligned}\right.
$$

and by positiveness of values $\mathrm{e}^{-\rho t}$ and $\alpha\left(1-y_{*}(t)\right) y_{*}(t)$, the relationships (16), (17) are transformed as follows

$$
\begin{gathered}
u_{*}(t)=\left\{\begin{array}{lll}
0, & \text { if } & \eta_{*}(t)<0, \\
\forall u \in[0,1], & \text { if } & \eta_{*}(t)=0, \\
1, & \text { if } & \eta_{*}(t)>0,
\end{array}\right. \\
v_{*}(t)=\left\{\begin{array}{lll}
0, & \text { if } & \eta_{*}(t)<\lambda, \\
\forall v \in\left[0, v_{\max }\right], & \text { if } & \eta_{*}(t)=\lambda, \\
v_{\max }, & \text { if } & \eta_{*}(t)>\lambda .
\end{array}\right.
\end{gathered}
$$

Therefore, based on systems (10), (18) and relationships (19), (20) we obtain the two-point boundary value problem (TPBVP) for the maximum principle of the type

$$
\begin{aligned}
& \left\{\begin{array}{l}
\dot{y}(t)=\alpha u(\eta(t))(1-y(t)) y(t)+v(\eta(t))-\delta y(t) \\
\dot{\eta}(t)=-(\alpha u(\eta(t))(1-2 y(t))-(\delta+\rho)) \eta(t)-(\alpha(1-2 y(t))-(\delta+\rho+\beta))
\end{array}\right. \\
& y(0)=y_{0}, \quad \eta(T)=0, \quad y_{0} \in(0,1)
\end{aligned}
$$

where

$$
\begin{gathered}
u(\eta)=\left\{\begin{array}{lll}
0, & \text { if } & \eta<0, \\
\forall u \in[0,1], & \text { if } & \eta=0, \\
1, & \text { if } & \eta>0,
\end{array}\right. \\
v(\eta)=\left\{\begin{array}{lll}
0, & \text { if } & \eta<\lambda, \\
\forall v \in\left[0, v_{\max }\right], & \text { if } & \eta=\lambda, \\
v_{\max }, & \text { if } & \eta>\lambda .
\end{array}\right.
\end{gathered}
$$

Note that the optimal controls $\left(u_{*}(t), v_{*}(t)\right)$, optimal solution $y_{*}(t)$ and corresponding solution $\eta_{*}(t)$ of the adjoint system (18) satisfy this boundary value problem. Next, we study the TPBVP for the maximum principle (21)-(24) in details. 


\section{Singular regimes}

Now, we will study the presence of singular regimes in the TPBVP (21)-(24). This means studying the occurrence of the cases in which the solution $\eta(t)$ can be equal to 0 or $\lambda$ on some finite subintervals of the segment $[0, T]$, or which means that the relationships (23), (24) cannot uniquely determine the controls $u$ and $v$, respectively.

Analyzing the formulas (23), (24) for the controls $u(\eta), v(\eta)$ we see that singular regimes are possible only in the following two situations.

- Let $\eta(t)=0$ for all $t \in \Delta \subset[0, T]$. Since $\eta(t)=0<\lambda$, then obviously that the control $v(\eta(t))=0$. From the equality $\eta(t)=0$ on the interval $\Delta$, we find that $\dot{\eta}(t)=0$. Substituting these equalities into the second equation of the TPBVP (21)-(24) we find the expression

$$
\alpha(1-2 y)-(\delta+\rho+\beta)=0 .
$$

By this equality, we introduce the value

$$
y_{\star}=\frac{\alpha-\delta-\rho-\beta}{2 \alpha} .
$$

Therefore, at the point $\left(y_{\star}, 0\right)$ of the plane $(y, \eta)$, the singular regime is possible only if $y_{\star}>0$, that also can be written as $\alpha>\delta+\rho+\beta$. If we have the opposite inequality $\alpha \leq \delta+\rho+\beta$, then the singular regime is not available.

Further, we suppose that $\alpha>\delta+\rho+\beta$. By the formula (25), we have $y_{\star} \in(0,1)$. Let us find the control $u$ on this singular regime. From the first equation of the TPBVP (21)-(24) we find the required formula

$$
u_{\text {sing }}=\frac{\delta}{\alpha\left(1-y_{\star}\right)} .
$$

It is easy to check that this control is admissible, i.e. $u_{\text {sing }} \in(0,1)$.

- Let $\eta(t)=\lambda$ for all $t \in \Delta \subset[0, T]$. Since $\eta(t)=\lambda>0$, then obviously that the control $u(\eta(t))=1$. From equality $\eta(t)=\lambda$ on the interval $\Delta$ we find that $\dot{\eta}(t)=0$. Substituting these equalities into the second equation of the TPBVP (21)-(24) we find the expression

$$
(\alpha(1-2 y)-(\delta+\rho)) \lambda+(\alpha(1-2 y)-(\delta+\rho+\beta))=0 .
$$


By this equality, introduce the value

$$
y_{\star \star}=\frac{\alpha-\delta-\rho-\beta(1+\lambda)^{-1}}{2 \alpha} .
$$

Therefore, at the point $\left(y_{\star \star}, \lambda\right)$ of the plane $(y, \eta)$, the singular regime is possible only if $y_{\star \star}>0$, or for $\alpha>\delta+\rho+\beta(1+\lambda)^{-1}$. If we have the opposite inequality $\alpha \leq \delta+\rho+\beta(1+\lambda)^{-1}$, then the singular regime is not possible.

Further, we suppose that $\alpha>\delta+\rho+\beta(1+\lambda)^{-1}$. By the formula (26), we have $y_{\star \star} \in(0,1)$. Let us find the control $v$ on this singular regime. From the first equation of the TPBVP (21)-(24) we find the required formula

$$
v_{\text {sing }}=\alpha y_{\star \star}^{2}-(\alpha-\delta) y_{\star \star} .
$$

It is easy to check that $v_{\text {sing }}<0$, and hence this control is not admissible.

Therefore, the singular regime:

$$
u=u_{\text {sing }}, \quad v=0, \quad y=y_{\star}, \quad \eta=0
$$

is possible, if the inequality $\alpha>\delta+\rho+\beta$ holds.

\section{Auxiliary results}

Let us consider the system of the equations (21) and the relationships (23), (24), which form the corresponding Hamiltonian system. We will investigate this system in details.

First, we will study the curves in the plane $(y, \eta)$ on which $\dot{y}=0$ and $\dot{\eta}=0$.

- Let $\dot{y}=0$. From the first equation of the system (21) we have

$$
\alpha u(\eta)(1-y) y+v(\eta)-\delta y=0 .
$$

Next, we consider the possible cases for the controls $u(\eta)$ and $v(\eta)$.

- Let $u(\eta)=0$. This is possible, when $\eta<0$. Then, $\eta<\lambda$, and therefore $v(\eta)=0$. Hence, we obtain the line $y=0$.

- Let $u(\eta)=1$. This is possible, when $\eta>0$. In this case there are two possible situations. 
* Let $\eta>\lambda$. Then, we have $v(\eta)=v_{\max }$. Therefore, the equation (28) can be written as

$$
\alpha y^{2}-(\alpha-\delta) y-v_{\max }=0 .
$$

The discriminant of this quadratic equation is positive, and so it has two distinct roots:

$$
\begin{aligned}
& \widetilde{y}=\frac{(\alpha-\delta)+\sqrt{(\alpha-\delta)^{2}+4 \alpha v_{\max }}}{2 \alpha}, \\
& \widetilde{\widetilde{y}}=\frac{(\alpha-\delta)-\sqrt{(\alpha-\delta)^{2}+4 \alpha v_{\max }}}{2 \alpha} .
\end{aligned}
$$

It is easy to check that $\widetilde{y} \in(0,1)$ and $\widetilde{\widetilde{y}}<0$. Then, we have the line $y=\widetilde{y}$.

* Let $0<\eta<\lambda$. Then, we have $v(\eta)=0$. Therefore, the equation (28) can be written as

$$
\alpha y^{2}-(\alpha-\delta) y=0,
$$

from which we find two roots $y=0$ and $y=\alpha^{-1}(\alpha-\delta)$. Introduce the value

$$
\bar{y}=\frac{\alpha-\delta}{\alpha} .
$$

The location of $\bar{y}$ on the axis $y$ depends on the ratio between $\alpha$ and $\delta$. It is easy to see that

- for $\alpha>\delta$ we have $\bar{y} \in(0,1)$;

- for $\alpha=\delta$ we have $\bar{y}=0$;

- for $\alpha<\delta$ we have $\bar{y}<0$.

Note immediately that for all values of the parameters the inequality $\bar{y}<\widetilde{y}$ holds. Finally, we obtain the line $y=0$ for $\alpha \leq \delta$, and the lines $y=0, y=\bar{y}$ for $\alpha>\delta$.

Combining the previous results for the curve $\dot{y}=0$, we have the relationships:

$$
\begin{gathered}
\alpha \leq \delta:\left\{\begin{array}{lll}
y=0, & \text { if } & \eta<\lambda, \\
y=\widetilde{y}, & \text { if } & \eta>\lambda,
\end{array}\right. \\
\alpha>\delta:\left\{\begin{array}{lll}
y=0, & \text { if } & \eta<\lambda, \\
y=\bar{y}, & \text { if } & 0<\eta<\lambda, \\
y=\widetilde{y}, & \text { if } & \eta>\lambda .
\end{array}\right.
\end{gathered}
$$


- Let $\dot{\eta}=0$. From the second equation of the system (21) we have

$$
(\alpha u(\eta)(1-2 y)-(\delta+\rho)) \eta+(\alpha(1-2 y)-(\delta+\rho+\beta))=0 .
$$

Next, we consider the possible cases for the control $u(\eta)$.

- Let $u(\eta)=1$. This is possible, when $\eta>0$. Introduce the value

$$
\widehat{y}=\frac{\alpha-\rho-\delta}{2 \alpha} \text {. }
$$

Then, from the expression (30) we find the formula

$$
\eta=-1-\frac{\beta}{2 \alpha(y-\widehat{y})} .
$$

The graph of the function (31) defines the increasing hyperbola with the line $y=\widehat{y}$ as its vertical asymptote. The location of $\widehat{y}$ on the axis $y$ depends on the ratio between $\alpha$ and $(\rho+\delta)$. It is easy to see that

$*$ for $\alpha>\rho+\delta$ we have $\widehat{y} \in(0,1)$;

$*$ for $\alpha=\rho+\delta$ we have $\widehat{y}=0$;

$*$ for $\alpha<\rho+\delta$ we have $\widehat{y}<0$.

Note immediately that the inequality $\widehat{y}<\widetilde{y}$ holds for all values of the parameters. Moreover, it is easy to see that for $\alpha \geq \delta$ the inequality $\widehat{y}<\bar{y}$ is valid. The graph of (31) is also characterized by the point $\left(y_{\star}, 0\right)$, in which this graph intersects with the axis $y$. From the formula (25) we find that the location of $y_{\star}$ on the axis $y$ depends on the ratio between $\alpha$ and $(\rho+\delta+\beta)$. It is easy to see that

$*$ for $\alpha>\rho+\delta+\beta$ we have $y_{\star} \in(0,1)$;

$*$ for $\alpha=\rho+\delta+\beta$ we have $y_{\star}=0$;

$*$ for $\alpha<\rho+\delta+\beta$ we have $y_{\star}<0$.

From the analysis of the formula (31) we obtain that for $\alpha \leq \rho+\delta$ there is no curve $\dot{\eta}=0$ at the considered region $\eta>0$. On the contrary, for $\alpha>\rho+\delta$ there exists the curve that is defined on the interval $\left(\max \left\{0 ; y_{\star}\right\}, \widehat{y}\right)$ and given by the formula (31).

- Let $u(\eta)=0$. This is possible, if $\eta<0$. Then, from the expression (30) we find the formula

$$
\eta=-\frac{2 \alpha}{\delta+\rho}\left(y-y_{\star}\right)
$$


which defines the decreasing linear function. As in the previous case, at the point $\left(y_{\star}, 0\right)$ the graph of the function (32) intersects with the axis $y$. Therefore, we obtain the curve $\dot{\eta}=0$ that is defined on the interval $\left(\max \left\{0 ; y_{\star}\right\}, 1\right)$ and given by the formula (32).

Combining the previous results for the curve $\dot{\eta}=0$, we have the relationships:

$$
\begin{gathered}
\alpha \leq \rho+\delta: \eta=-\frac{2 \alpha}{\delta+\rho}\left(y-y_{\star}\right), \text { if } \quad y \in(0,1), \\
\rho+\delta<\alpha \leq \rho+\delta+\beta: \begin{cases}\eta=-\frac{2 \alpha}{\delta+\rho}\left(y-y_{\star}\right), & \text { if } \quad y \in(0,1), \\
\eta=-1-\frac{\beta}{2 \alpha(y-\widehat{y})}, & \text { if } \quad y \in(0, \widehat{y}),\end{cases} \\
\alpha>\rho+\delta+\beta: \begin{cases}\eta=-\frac{2 \alpha}{\delta+\rho}\left(y-y_{\star}\right), & \text { if } \quad y \in\left(y_{\star}, 1\right), \\
\eta=0, & \text { if } \quad y=y_{\star}, \\
\eta=-1-\frac{\beta}{2 \alpha(y-\widehat{y})}, & \text { if } \quad y \in\left(y_{\star}, \widehat{y}\right) .\end{cases}
\end{gathered}
$$

Secondly, we define the signs of derivatives $\dot{y}$ and $\dot{\eta}$ in the plane $(y, \eta)$.

- Let us consider the derivative $\dot{y}$. For this, we transform the first equation of the system (21) as follows

$$
\dot{y}=-\alpha u(\eta) y^{2}+(\alpha u(\eta)-\delta) y+v(\eta) .
$$

From the analysis of the expression (36), we see that

- at $\eta<0$ the equalities $u(\eta)=0, v(\eta)=0$ and the relationship $\dot{y}=-\delta y<0$ hold. The line $y=0$ itself is a trajectory of the Hamiltonian system (21), (23), (24);

- at $\eta>\lambda$ the equalities $u(\eta)=1, v(\eta)=v_{\max }$ and the relationship $\dot{y}=-\alpha(y-\widetilde{y})(y-\widetilde{\widetilde{y}})$ hold. From this expression we conclude that to the right of the line $y=\widetilde{y}$ the inequality $\dot{y}<0$ holds, and to the left of it the inequality $\dot{y}>0$ is valid. The line $y=\widetilde{y}$ itself is a trajectory of the Hamiltonian system (21), (23), (24);

- at $0<\eta<\lambda$ the equalities $u(\eta)=1, v(\eta)=0$ and the relationship $\dot{y}=-\alpha y(y-\bar{y})$ hold. From this expression we conclude that for $\alpha \leq \delta$ the inequality $\dot{y}<0$ is valid. For $\alpha>\delta$ we obtain that to the right of the line $y=\bar{y}$ the inequality $\dot{y}<0$ holds, and to the left of it the inequality $\dot{y}>0$ is valid. The lines $y=0, y=\bar{y}$ themselves are the trajectories of the Hamiltonian system (21), (23), (24). 
- Let us consider the derivative $\dot{\eta}$. For this, we will transform the second equation of the system (21)

- for $\eta<0$ to the type

$$
\dot{\eta}=(\delta+\rho)\left(\eta+\frac{2 \alpha}{\delta+\rho}\left(y-y_{\star}\right)\right),
$$

- for $\eta>0$ to the type

$$
\dot{\eta}=2 \alpha(y-\widehat{y})\left(\eta+1+\frac{\beta}{2 \alpha(y-\widehat{y})}\right) .
$$

Analyzing the signs of the terms in the right hand sides of the equations (37), (38) in the regions $\eta<0, \eta>0$, we have the following conclusions:

- at $\alpha \leq \delta+\rho$ above the line (33) we see that $\dot{\eta}>0$, and below this line we have $\dot{\eta}<0$;

- at $\rho+\delta<\alpha \leq \rho+\delta+\beta$ to the left of the curves (34) we see that $\dot{\eta}<0$, and to the right of these curves we have $\dot{\eta}>0$;

- at $\alpha>\rho+\delta+\beta$ to the left of the curve (35) we see that $\dot{\eta}<0$, and to the right of this curve we have $\dot{\eta}>0$.

\section{Hamiltonian system}

Now, we will analyze the Hamiltonian system (21), (23), (24) depending on the initial condition $y_{0}$ and the parameters $\alpha, \delta, \rho, \beta$. The following three cases will be considered.

Case 1. Let $\alpha<\delta+\rho+\beta$. It follows from the arguments of Section 5 that the Hamiltonian system (21), (23), (24) has no rest points in the region $\eta>0$. At $\eta<0$ the system (21) takes the form of the linear system

$$
\left\{\begin{array}{l}
\dot{y}=-\delta y \\
\dot{\eta}=2 \alpha y+(\delta+\rho)\left(\eta-\frac{2 \alpha y_{\star}}{\delta+\rho}\right) .
\end{array}\right.
$$

Analysis of this system shows that there exists a rest point

$$
\left(0, \frac{2 \alpha y_{\star}}{\delta+\rho}\right), y_{\star}<0 \text {. }
$$


The eigenvalues $(-\delta),(\delta+\rho)$ of the system (39) are real and have opposite signs. Therefore, the rest point (40) is a saddle point. The line $y=0$ from the relationships (29) corresponds to the positive eigenvalue, and the line

$$
\eta=-\frac{2 \alpha}{2 \delta+\rho}\left(y-\frac{2 \delta+\rho}{\delta+\rho} y_{\star}\right)
$$

to the negative eigenvalue. Moreover, both of these lines are the trajectories of the system (39). The behavior of the trajectories similar to this system is presented, for example, in [14]. Considering the signs of the derivatives $\dot{y}$ and $\dot{\eta}$ it is easy to see that the trajectories of the Hamiltonian system (21), (23), (24) with growth of $t$ will approach the line $\eta=0$ only from the region $\eta<0$, intersect it, and then go away from it in the region $\eta>0$. By the second initial condition (22), the solution of the TPBVP (21)-(24) must end on the line $\eta=0$, and hence, by the relationships (23), (24), the corresponding controls $(u(\eta), v(\eta))$ have the type

$$
u(\eta)=0, \quad v(\eta)=0 .
$$

In the considered case at $\alpha>\delta+\rho+\beta(1+\lambda)^{-1}$, in the region $\eta>0$ there is one more rest point

$$
\left(0,-\frac{y_{\star}}{\widehat{y}}\right), y_{\star}<0
$$

Simple calculations show that the matrix of the linear system corresponding to the system $(21)$ has the eigenvalues $(\alpha-\delta),(-2 \alpha \widehat{y})$ that are real and have opposite signs. Therefore, the rest point (42) is unstable. The behavior of trajectories of the Hamiltonian system (21) in the neighborhood of the point (42) does not affect their behavior in the neighborhood of the line $\eta=0$. In this situation the phase portrait of the Hamiltonian system (21), (23), (24) is presented in Figure 1.

Case 2. Let $\alpha=\delta+\rho+\beta$. The difference from the previous case is only that in this case, the rest points (40), (42) are converted into one point which is located at the origin. The behavior of the trajectories of the Hamiltonian system (21), (23), (24) in the neighborhood of the line $\eta=0$ is similar to Case 1 . Therefore, the controls $(u(\eta), v(\eta))$ have the types (41).

Case 3. Let $\alpha>\delta+\rho+\beta$. We see that $y_{\star}>0$. It follows from the arguments of Section 5 that to the right of the curve (35), the behavior of the trajectories of the Hamiltonian system (21), (23), (24) is the same as in the previous two cases. Therefore, at $y_{0}>y_{\star}$ with growth of $t$, the trajectories approach the line $\eta=0$ from the region $\eta<0$, intersect it, and then go away from it in the region $\eta>0$. So, the controls $(u(\eta), v(\eta))$ have the types (41). Moreover, the singular regime (27) is possible if and only if the motion occurs along the line (32) and ends at the point $\left(y_{\star}, 0\right)$. (In Figure 2 such a trajectory is shown as a thick curve that 




Figure 1: Phase portrait of the Hamiltonian system at $\alpha<\delta+\rho+\beta$.

starts on the right of $y_{\star}$ and at negative value of $\eta$ ). The corresponding control $u(\eta)$ switches from the value 0 to the value $u_{\text {sing }}$, and the control $v(\eta)$ remains equal to the value 0 . At $y_{0}=y_{\star}$ the controls $(u(\eta), v(\eta))$ have the singular regime (27), i. e. the control $(u(\eta), v(\eta))$ have the types

$$
u(\eta)=u_{\text {sing }}, \quad v(\eta)=0 .
$$

At last, we can show that at the region $\eta>0$ there exists the trajectory of the Hamiltonian system (21), (23), (24) starting from the point $\left(y_{\star}, 0\right)$ and moving to the axis $\eta$ as $t$ is decreasing. Therefore, to the left of the curve (35), the behavior of the trajectories of the system (21) is opposite. Namely, at $y_{0}<y_{\star}$ with the growth of $t$, the trajectories approach the line $\eta=0$ from the region $\eta>0$, intersect it, and then go away from it in the region $\eta<0$. Therefore, by the relationship (23), we obtain $u(\eta)=1$, and the control $v(\eta)$ will be either 0 or it will switch from the value $v_{\max }$ to 0 . Also, when the motion occurs alone the mentioned trajectory and ends at the point $\left(y_{\star}, 0\right)$, then the singular regime (27) may occur. The corresponding control $v(\eta)$ remains at the value 0 , and the

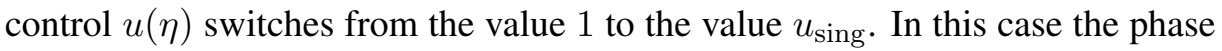
portrait of the Hamiltonian system (21), (23), (24) is presented in Figure 2. 


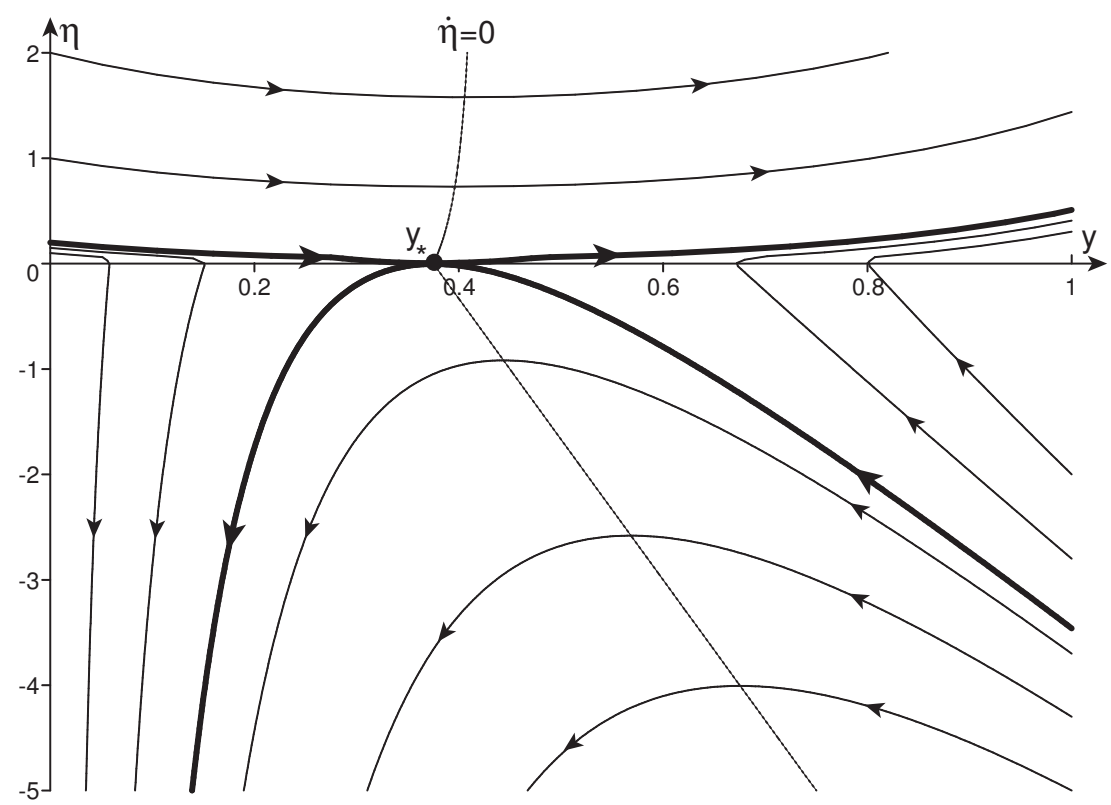

Figure 2: Phase portrait of the Hamiltonian system at $\alpha>\delta+\rho+\beta$.

Remark 1 Similar analysis of Hamiltonian systems for economic growth models is presented in [9, 2, 6].

\section{Types of the optimal controls}

From the arguments of Section 6 we can make the following conclusions about the types of the optimal controls $\left(u_{*}(t), v_{*}(t)\right), t \in[0, T]$. Depending on the initial condition $y_{0}$ and the parameters $\alpha, \delta, \rho, \beta$ the following situations are possible.

- Let $\alpha \leq \delta+\rho+\beta$. Then, the optimal controls $\left(u_{*}(t), v_{*}(t)\right)$ are the functions of the types

$$
u_{*}(t)=0, \quad v_{*}(t)=0, \quad t \in[0, T] .
$$

- Let $\alpha>\delta+\rho+\beta$. Then,

- if $y_{0}>y_{\star}$, the optimal controls $\left(u_{*}(t), v_{*}(t)\right)$ can be the functions 
either of the types

$$
u_{*}(t)=0, \quad v_{*}(t)=0, \quad t \in[0, T],
$$

or

$$
u_{*}(t)=\left\{\begin{array}{ll}
0, & \text { if } \quad 0 \leq t \leq \theta_{*}, \\
u_{\text {sing }}, & \text { if } \quad \theta_{*}<t \leq T,
\end{array} \quad v_{*}(t)=0, \quad t \in[0, T],\right.
$$

where $\theta_{*} \in(0, T)$ is the moment of switching;

- if $y_{0}=y_{\star}$, the optimal controls $\left(u_{*}(t), v_{*}(t)\right)$ are the functions of the types

$$
u_{*}(t)=u_{\text {sing }}, \quad v_{*}(t)=0, \quad t \in[0, T]
$$

- if $y_{0}<y_{\star}$, the optimal controls $\left(u_{*}(t), v_{*}(t)\right)$ can be the functions either of the types

$$
u_{*}(t)=1, \quad v_{*}(t)=0, \quad t \in[0, T],
$$

or

$$
u_{*}(t)=1, \quad t \in[0, T], \quad v_{*}(t)= \begin{cases}v_{\max }, & \text { if } 0 \leq t \leq \tau_{*}, \\ 0, & \text { if } \tau_{*}<t \leq T,\end{cases}
$$

or

$$
u_{*}(t)=\left\{\begin{array}{ll}
1, & \text { if } \quad 0 \leq t \leq \theta_{*}, \\
u_{\text {sing }}, & \text { if } \quad \theta_{*}<t \leq T,
\end{array} \quad v_{*}(t)=0, \quad t \in[0, T],\right.
$$

or

$u_{*}(t)=\left\{\begin{array}{ll}1, & \text { if } 0 \leq t \leq \theta_{*}, \\ u_{\mathrm{sing}}, & \text { if } \theta_{*}<t \leq T,\end{array} v_{*}(t)= \begin{cases}v_{\max }, & \text { if } 0 \leq t \leq \tau_{*}, \\ 0, & \text { if } \tau_{*}<t \leq T\end{cases}\right.$

where $\theta_{*}, \tau_{*} \in(0, T)$ are the moments of switching.

In Figures 3-9 the graphs of the optimal controls $\left(u_{*}(t), v_{*}(t)\right)$ are presented for the situation $\alpha>\delta+\rho+\beta$ at different values of $y_{0}$, respectively. 

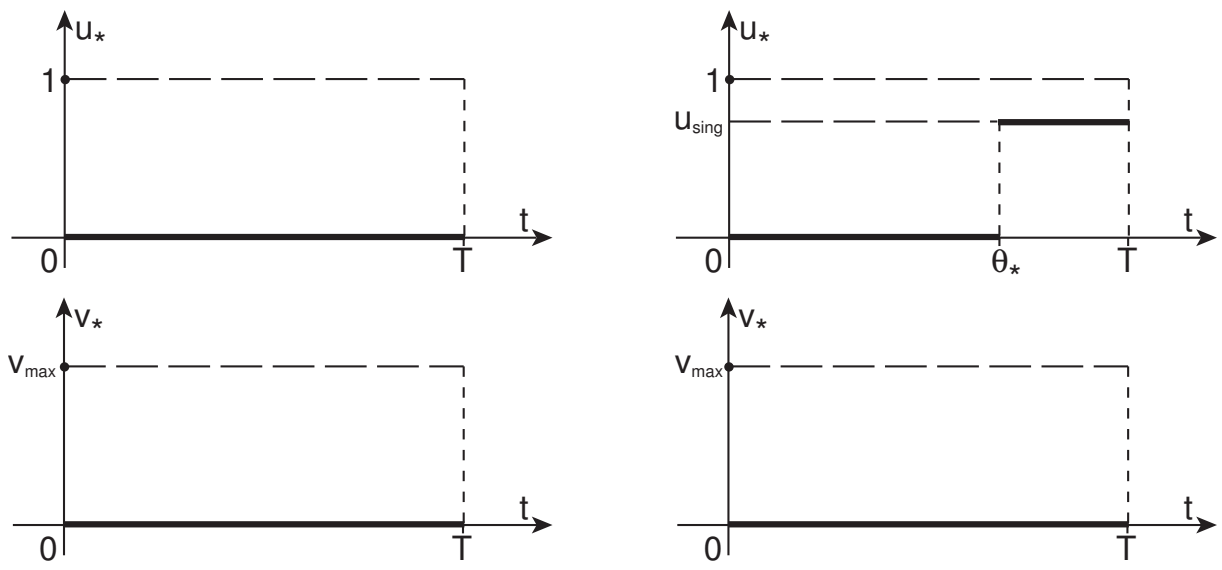

Figure 3: Optimal controls $\left(u_{*}(t), v_{*}(t)\right) \quad$ for $y_{0}>y_{\star}$.

Figure 4: Optimal controls $\left(u_{*}(t), v_{*}(t)\right) \quad$ for $y_{0}>y_{\star}$.
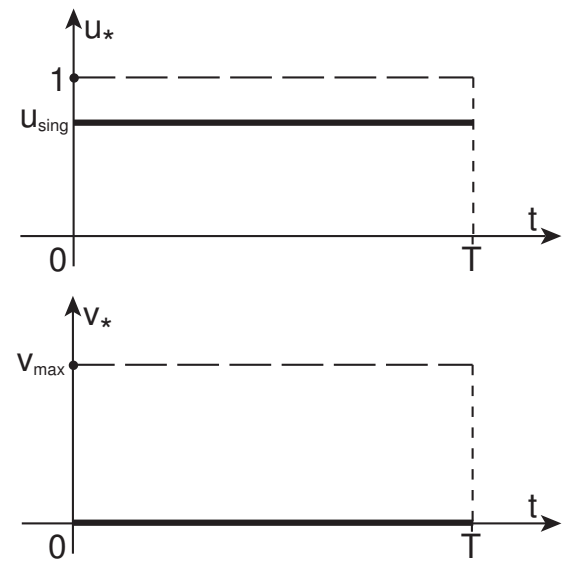

Figure 5: Optimal controls $\left(u_{*}(t), v_{*}(t)\right)$ for $y_{0}=y_{\star}$. 

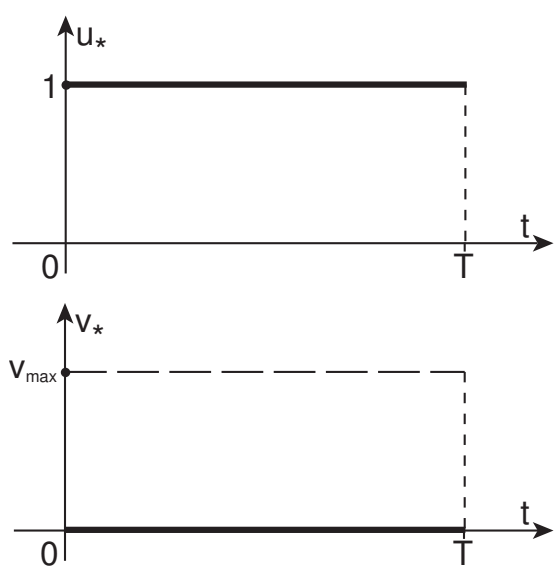

Figure 6: Optimal controls $\left(u_{*}(t), v_{*}(t)\right)$ for $y_{0}<y_{\star}$.
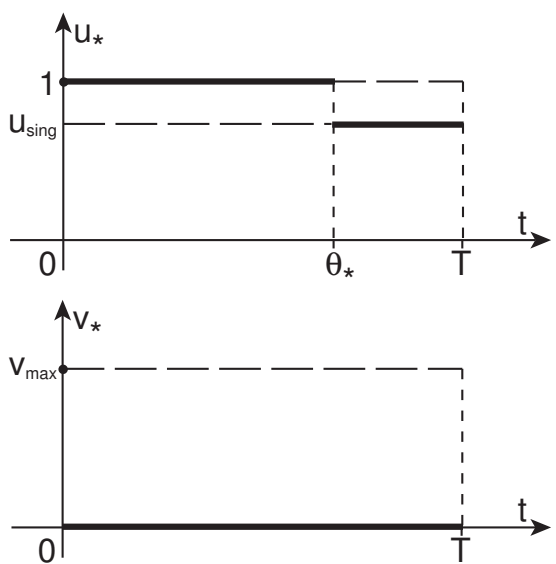

Figure 8: Optimal controls $\left(u_{*}(t), v_{*}(t)\right) \quad$ for
$y_{0}<y_{\star}$.
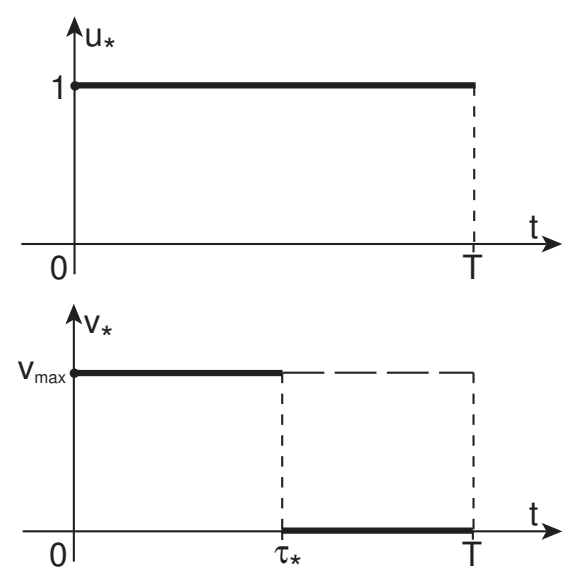

Figure 7: Optimal controls $\left(u_{*}(t), v_{*}(t)\right)$ for $y_{0}<y_{\star}$.
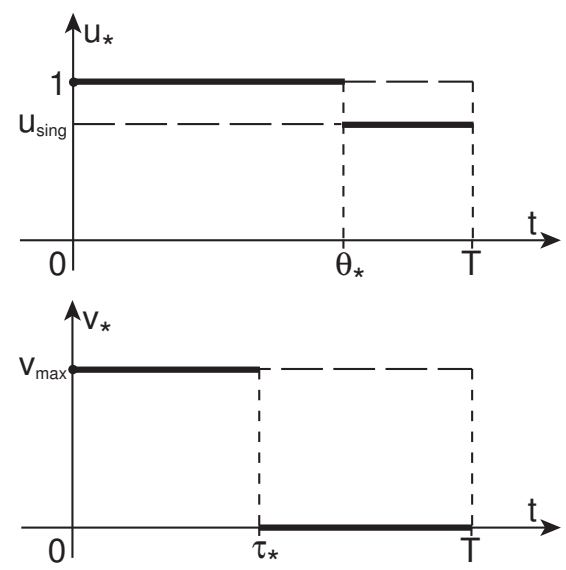

Figure 9: Optimal controls $\left(u_{*}(t), v_{*}(t)\right) \quad$ for
$y_{0}<y_{\star}$. 


\section{Numerical solution of the optimal control problem}

Now, we describe the method of solving the optimal control problem (10), (12)(14) for different cases.

- Let $\alpha>\delta+\rho+\beta$ and $y_{0}>y_{\star}$. For an arbitrary value $\theta \in[0, T]$ we define the control $u_{\theta}(t)$ as

$$
u_{\theta}(t)= \begin{cases}0, & \text { if } \quad 0 \leq t \leq \theta \\ u_{\text {sing }}, & \text { if } \quad \theta<t \leq T\end{cases}
$$

The controls $\left(u_{\theta}(t), v_{0}(t)\right)$, where $v_{0}(t)=0$, include all possible types (44), (45) of the optimal controls $\left(u_{*}(t), v_{*}(t)\right)$. Then, we substitute the controls $\left(u_{\theta}(t), v_{0}(t)\right)$ into the system (10) and integrate this system over the interval $[0, T]$. This yields the function $y_{\theta}(t)$, which corresponds to the controls $\left(u_{\theta}(t), v_{0}(t)\right)$, and which we then substitute into the functional (13). The result is the function of one variable

$$
F(\theta)=J\left(u_{\theta}, v_{0}\right), \quad \theta \in[0, T] .
$$

- Let $\alpha>\delta+\rho+\beta$ and $y_{0}<y_{\star}$. For arbitrary values $\theta, \tau \in[0, T]$ we define the controls $u_{\theta}(t), v_{\tau}(t)$ as

$$
u_{\theta}(t)=\left\{\begin{array}{ll}
1, & \text { if } 0 \leq t \leq \theta, \\
u_{\text {sing }}, & \text { if } \theta<t \leq T,
\end{array} \quad v_{\tau}(t)=\left\{\begin{array}{lll}
v_{\max }, & \text { if } & 0 \leq t \leq \tau \\
0, & \text { if } & \tau<t \leq T .
\end{array}\right.\right.
$$

The controls $\left(u_{\theta}(t), v_{\tau}(t)\right)$ include all possible types (47)-(50) of the optimal controls $\left(u_{*}(t), v_{*}(t)\right)$. Then, we substitute the controls $\left(u_{\theta}(t), v_{\tau}(t)\right)$ into the system (10) and integrate this system over the interval $[0, T]$. This yields the function $y_{\theta, \tau}(t)$, which corresponds to the controls $\left(u_{\theta}(t), v_{\tau}(t)\right)$, and which we then substitute into the functional (13). The result is the function of two variables

$$
G(\theta, \tau)=J\left(u_{\theta}, v_{\tau}\right), \quad \theta, \tau \in[0, T] .
$$

Thus, the optimal control problem (10), (12)-(14) is reduced to the problems of constrained minimization:

$$
F(\theta) \rightarrow \min _{\theta \in[0, T]}
$$

for $\alpha>\delta+\rho+\beta$ and $y_{0}>y_{\star}$

$$
G(\theta, \tau) \rightarrow \min _{(\theta, \tau) \in[0, T] \times[0, T]}
$$


for $\alpha>\delta+\rho+\beta$ and $y_{0}<y_{\star}$. The methods for numerical solution to these problems are well-known [17].

In the situations $\alpha \leq \delta+\rho+\beta, \alpha>\delta+\rho+\beta$ and $y_{0}=y_{\star}$, the optimal controls $\left(u_{*}(t), v_{*}(t)\right)$ are the constant functions of the types (43), (46) respectively, and therefore, they do not require numerical calculations.

\section{Discussion of future research}

Further investigations of the model presented in Section 2 can be conducted not only by considering the Cobb-Douglas production function of the general form, i.e. $\sigma \in(0,1)$, but also it can proceed in the following two ways.

First, we can consider a nonlinear model of the market, if instead of the demand formula (5) we study the dependence of the type

$$
p=\bar{p}\left(1-\left(\frac{b Y}{\bar{p}}\right)^{\frac{1}{\gamma}}\right),
$$

where $\gamma>0, \gamma \neq 1$ is a given constant. Obviously, the expression (5) occurs, if $\gamma=1$. The formula (51) reflects the effects of price dropping or sharp price increase of the consumer good on the market.

Secondly, we can consider a situation, when not all produced consumer good $Y$ is sent to the market, but only its part, $w Y$. Here $w \in[0,1]$. The remaining part $(1-w) Y$ is sent to the company's warehouse for storage. In this case, it is possible to control of these parts. Therefore, there is another control $w(t)$, $t \in[0, T]$ satisfying the restrictions $0 \leq w(t) \leq 1$. Then the Cauchy problem (6) and the objective function (7) will have the following types:

$$
\begin{aligned}
& \left\{\begin{array}{l}
\dot{x}(t)=u(t) w(t)(\bar{p}-b w(t) \Pi(x(t))) \Pi(x(t))+K(t)-\delta x(t), \quad t \in[0, T], \\
x(0)=x_{0}, x_{0}>0 .
\end{array}\right. \\
& I(u, w, K)=\int_{0}^{T} \mathrm{e}^{-\rho t}\left\{(1-u(t)) w(t)(\bar{p}-b w(t) \Pi(x(t))) \Pi(x(t))-p_{0} \Pi(x(t))\right. \\
& \quad-q(1-w(t)) \Pi(x(t))-(1+\lambda) K(t)\} d t+\mathrm{e}^{-\rho T} x(T) .
\end{aligned}
$$

where $q$ is storage cost of a unit of the consumer good.

\section{Conclusion}

In this paper we obtained important mathematical results regarding optimal production and sales activity of a monopoly. We found that the optimal strategies 
depend on the comparison of the values of the model's parameters, and that depending on whether $\alpha$ is greater or less than or equal to the value of $(\delta+\rho+\beta)$, different optimal controls can be used in order to maximize profit. It is interesting that for some initial conditions and model's parameter, singular control can be also optimal. Let us rewrite the key comparison $\alpha$ vs $(\delta+\rho+\beta)$ in the following equivalent form $\left(\bar{p}-p_{0}\right) r$ vs $(\delta+\rho)$. On its left, there is the profit of the monopoly obtained from the sales of the consumer good on the market at the maximum possible market price $\bar{p}$ produced by the unit of the production funds. On its right, there is the total loss of the company caused by the amortization of the production funds $(\delta)$ and by rate of inflation $(\rho)$. Thus, if the profit $\left(\bar{p}-p_{0}\right) r$ is greater than the total loss $(\delta+\rho)$, then depending on the initial condition $y_{0}$, the company can do both, reinvest part of the obtained profit into expansion of the production, and even take loans. Opposite, if $\left(\bar{p}-p_{0}\right) r \leq \delta+\rho$, then the monopoly must be advised to produce and sale without taking any loans or planning expansion of the production. Finally, investigation of the modified model with nonlinear production function and with additional inventory control will be conducted in our future research.

\section{Acknowledgements}

The authors would like to thank Prof. S.M. Aseev for his suggestions regarding the statement of the problem and Dr. S.N. Avvakumov for his help in creating illustrations for this paper.

\section{References}

[1] Arrow, K.J. (1968) "Application of control theory to economic growth", in: Lectures in Applied Mathematics, Mathematics of the Decision Sciences, part 2, 12, AMS, Providence, RI: 85-119.

[2] Aseev, S.M.; Kryazhimskii, A.V. (2007) "The Pontryagin maximum principle and optimal economic growth problems", Proceedings of the Steklov Institute of Mathematics 257(1): 1-255.

[3] Caputo, M.R. (2005) Foundations of Dynamic Economic Analysis: Optimal Control Theory and Applications. Cambridge University Press, Cambridge, UK.

[4] Carlson, D.A.; Haurie, A.B.; Leizarowitz, A. (1991) Infinite Horizon Optimal Control: Deterministic and Stochastic Systems. Springer-Verlag, Berlin-Heidelberg-New York. 
[5] Demin, N.S.; Kuleshova, E.V. (2008) "Control of a one-sector economy on finite time interval with account of tax deductions", Journal of Computer and Systems Sciences International 47(6): 918-929.

[6] Grigorieva, E.V.; Khailov, E.N. (2007) "Optimal control of a nonlinear model of economic growth", Discrete and Continuous Dynamical Systems, supplement volume: 456-466.

[7] Intriligator, M.D. (2002) Mathematical Optimization and Economic Theory. SIAM, Philadelphia.

[8] Khelifi, A. (2010) "Explicit solution to optimal growth models", International Journal of Economics and Finance 2(5): 116-121.

[9] Kryazhimskii, A.; Watanabe, C. (2004) Optimization of Technological Growth. Gendaitosho, Japan.

[10] Lee, E.B.; Marcus, L. (1967) Foundations of Optimal Control Theory. John Wiley \& Sons, New York.

[11] Leontief, W.W. (1966) Input-Output Economics. Oxford University Press, New York.

[12] Loon, P.A. (1983) Dynamic Theory of the Firm: Production, Finance, and Investment. Lecture Notes in Economics and Mathematical Systems, vol. 218. Springer-Verlag, Berlin-Heidelberg-New York.

[13] Pontryagin, L.S.; Boltyanskii, V.G.; Gamkrelidze, R.V.; Mishchenko, E.F. (1962) Mathematical Theory of Optimal Processes. John Wiley \& Sons, New York.

[14] Robinson, R.C. (2004) An Introduction to Dynamical Systems: Continuous and Discrete. Pearson Prentice Hall, New Jersey.

[15] Solow, R.M. (1956) "Contribution to the theory of economic growth", Quarterly Journal of Economics 70(1): 65-94.

[16] Swan, T.W. (1956) "Economic growth and capital accumulation", Economic Record 32(2): 334-361.

[17] Vasil'ev, F.P. (2002) Optimization Methods. Factorial Press, Moscow.

[18] Weitzman, M.L. (2003) Income, Wealth, and Maximum Principle. Harvard University Press, Cambridge, UK. 